\title{
The next battle line against melanoma
}

\section{By Lev Osherovich, Senior Writer}

Boston and Los Angeles researchers have painted the best portrait to date of the resistance mutations that arise from treating melanoma with BRAF and MEK inhibitors. ${ }^{1-4}$ Although the resistance pathways were known, the new information on the specific activating mutations could translate to improved drug combinations tailored for individual patients.

The melanoma space has undergone a dramatic shift in the last two years, as the long-standing first-line chemotherapeutic dacarbazine was supplanted following the 2012 approval of Zelboraf vemurafenib, a selective BRAF inhibitor from Roche, Chugai Pharmaceutical Co. Ltd. and Daiichi Sankyo Co. Ltd. The FDA approved a second BRAF inhibitor, GlaxoSmithKline plc's Tafinlar dabrafenib (GSK2118436), in 2013.

However, many patients receiving BRAF inhibitor monotherapy relapse within a year because residual tumor cells acquire mutations that upregulate prosurvival signaling pathways downstream of BRAF.

Indeed, in 2010, cancer genome profiling studies from independent teams led by Levi Garraway and Roger Lo helped build a case for inhibiting MAP kinase kinase 1 (MAP2K1; MEK1) and MAP2K2

\section{"They're starting with} patient samples to identify new mechanisms of resistance and are then working backward to fill in the pieces with laboratory cell lines. This is a great example of going from patients back to the lab and back again."

$$
\begin{array}{r}
\text {-Jeff Legos, } \\
\text { GlaxoSmithKline plc }
\end{array}
$$

(MEK2) to enhance the efficacy and therapeutic window of BRAF inhibitors. ${ }^{5,6}$ MEK1 and MEK2 are directly activated by BRAF.

Garraway is an assistant professor of medicine at Harvard Medical School and an associate professor of medicine at the DanaFarber Cancer Institute. Lo is an assistant professor of medicine at the University of California, Los Angeles.

The first MEK inhibitorMekinist trametinib (GSK1120212) from GSK and Japan Tobacco Inc.-was approved in May 2013 as monotherapy for melanomas with BRAF V600E and V600K mutations. This week, the FDA approved a combination of Mekinist and Tafinlar for unresectable or metastatic melanoma with a BRAF V600E or V600K mutation.

"BRAF and MEK inhibitors are being studied together in combination, and those trials are very promising and will be standard of care in the near term," said Lo. "Beyond that, we have to develop ways to keep the disease under control" because resistance to the combination does arise.

Indeed, patients who respond well to dual inhibition of BRAF and MEK can eventually relapse.

Previous cell culture and clinical studies suggested mutations that increase signaling through the MAPK and phosphoinositide 3-kinase (PI3K) pathways could counteract BRAF and MEK inhibition. ${ }^{7}$ Until now, however, the precise mechanisms by which resistance emerges were unclear.

The Lo and Garraway teams isolated specific MAPK and PI3K pathway mutations and amplifications in clinical samples from drugresistant tumors and characterized how these mutations alter cellular signaling to promote drug resistance.

"They're starting with patient samples to identify new mechanisms of resistance and are then working backward to fill in the pieces with laboratory cell lines," said Jeff Legos, oncology medicine development leader at GSK. "This is a great example of going from patients back to the lab and back again."

\section{Resistance MEKanisms}

Lo's team collaborated with Australian researchers to sequence the exomes and transcriptomes of 100 tumors from 44 patients who became resistant to BRAF inhibitors.

Garraway collaborated with Jennifer Wargo, an assistant professor of surgical oncology at The University of Texas MD Anderson Cancer Center, and a consortium of European researchers to analyze the genomes of 45 patients with acquired BRAF inhibitor resistance and 5 patients with tumors resistant to a combination of BRAF and MEK inhibitors.

By comparing the sequences with pretreatment biopsies, the teams mapped the mutations and genetic rearrangements likely to underlie drug resistance.

The bulk of the mutations led to overexpression or activation of known players in the BRAF and MAPK signaling pathways, such as MEK1 and MEK2.

The teams went on to culture patient-derived and engineered cell lines in vitro and used these cell culture models to study how resistancedriving mutations evolve.

Lo's team found that different drug-resistant tumors in the same patients carried a multitude of mutations and genetic rearrangements and showed different growth rates and drug sensitivities in vitro.

To Lo, this finding suggests that there are many possible routes for tumors to evade treatments that hit a single pathway, such as the BRAF and MEK inhibitor combination.

"Melanoma is a highly mutated cancer with lots of heterogeneity," said Lo. "There are so many possible mutations that if you treat with a single agent, it becomes much harder to treat when it comes back."

Results from both teams were reported in Cancer Discovery. Lo did not file for patents on his findings but has previously filed a patent on assays and therapies for BRAF-resistant melanoma. The licensing status of this patent is undisclosed.

Garraway did not disclose the IP status of his work. 


\section{AKT now}

It comes as no surprise that activating mutations in the MAPK pathway can lead to BRAF inhibitor resistance. However, the variety of mutations and gene amplifications uncovered by the two teams suggests that hitting MAPK signaling will be a game of whack-a-mole, with multiple means for tumors to evade targeted therapies.

Nevertheless, identifying specific resistance mechanisms in individual patients could help clinicians tailor second-line therapies.

For example, Garraway's team identified a subset of patients in which hitting targets downstream of MEK1 could be useful. The team reported that, in cultured tumors resistant to both BRAF and MEK1 inhibitors, tumor growth could be prevented with a tool compound that blocks MAP kinase 1 (MAPK1; ERK-2) and MAPK3 (ERK-1), a pair of kinases downstream of MEK1.

Last year, researchers at Merck \& Co. Inc. reported proof of concept for inhibiting ERK-1 and 2 in cultured melanoma tumors resistant to BRAF and MEK inhibitors. ${ }^{8}$

Aeterna Zentaris Inc. has the ERK-1 and 2 inhibitor AEZS-136 in preclinical development for cancer.

Meanwhile, Lo advocates hitting PI3K signaling, a proliferative pathway that cross-talks with MAPK signaling.

His team found that $22 \%$ of BRAF inhibitor-resistant tumors had elevated protein kinase $\mathrm{B}$ (PKB; PKBA; AKT; AKT1) signaling. AKT is a key component of the PI3K pathway.

In patient samples, Lo's team found two activating mutations in AKT that led to higher phosphoinositide levels and proliferative signaling than those seen in treatment-naïve cells.

In cell culture, combined pharmacological inhibition of BRAF and AKT decreased growth in cells with mutant AKT compared with vehicle.

Legos said that AKT or PI3K inhibitors could be especially useful in a subset of patients whose tumors are driven by loss of PTEN (MMAC1; TEP1), a tumor suppressor that regulates PI3K signaling.

"Where the AKT/PI3K pathway is most relevant is in patients with a PTEN deficiency, in which there are no brakes on the PI3K pathway. This is about $10 \%$ of patients, so there's an overlap between these patients and BRAF mutants," said Legos.

Lo said that his team is collaborating with GSK to test AKT inhibitor GSK2141795 in combination with Tafinlar in a Phase I/II trial in patients with BRAF-mutant melanoma.

He noted that $\mathrm{AKT}$ is an attractive target because AKT inhibitors are generally better tolerated than direct PI3K inhibitors.

Oncothyreon Inc. and Novartis AG each have their own PI3K inhibitors in Phase I/II trials in BRAF-mutant melanoma in combination with Zelboraf.

Lo and Legos think that the future of melanoma treatment will likely involve triple therapy of BRAF, MEK, and AKT and PI3K inhibitors.

"The clinical takeaway is that you want to inhibit as many of these targets or pathways as possible up front," said Legos. "A triple therapy is quite a reasonable approach."
Because combining all three compounds at the same time will likely pose tolerability challenges, Lo and Legos think that clinicians will likely cycle through pairwise combinations. The challenge will be to devise patient-specific combinations and dosing regimens in which drugs are swapped out before resistant cells become prevalent.

“There's some evidence for benefit from intermittent therapies with BRAF and MEK inhibitors. You can imagine rotating through different combinations over time in one patient," said Lo. "We have already proposed such a study and will start enrollment this year."

Osherovich, L. SciBX 7(1); doi:10.1038/scibx.2014.2

Published online Jan. 9, 2014

\section{REFERENCES}

1. Shi, H. et al. Cancer Discov.; published online Nov. 21, 2013; doi:10.1158/2159-8290.CD-13-0279

Contact: Roger S. Lo, University of California, Los Angeles, Calif. e-mail: rlo@mednet.ucla.edu

2. Shi, H. et al. Cancer Discov.; published online Nov. 21, 2013; doi:10.1158/2159-8290.CD-13-0642

Contact: Roger S. Lo, University of California, Los Angeles, Calif. e-mail: rlo@mednet.ucla.edu

3. Wagle, N. et al. Cancer Discov.; published online Nov. 21, 2013; doi:10.1158/2159-8290.CD-13-0631

Contact: Jennifer A. Wargo, Harvard Medical School, Boston, Mass.

e-mail: jwargo@mdanderson.org

Contact: Levi A. Garraway, Dana-Farber Cancer Institute, Boston, Mass.

e-mail: levi_garraway@dfci.harvard.edu

4. Van Allen, E.M. et al. Cancer Discov.; published online Nov. 21, 2013; doi:10.1158/2159-8290.CD-13-0617

Contact: Dirk Schadendorf, University Hospital Essen, Essen, Germany

e-mail: dirk.schadendorf@uk-essen.de

Contact: Levi A. Garraway, Dana-Farber Cancer Institute, Boston,

Mass.

e-mail: levi_garraway@dfci.harvard.edu

5. Nazarian, R. et al. Nature 468, 973-977 (2010)

6. Johannessen, C.M. et al. Nature 468, 968-972 (2010)

7. Villanueva, J. et al. Cell Rep. 4, 1090-1099 (2013)

8. Morris, E.J. et al. Cancer Discov. 3, 742-750 (2013)

\section{COMPANIES AND INSTITUTIONS MENTIONED}

Aeterna Zentaris Inc. (TSX:AEZ; NASDAQ:AEZS), Quebec City, Quebec, Canada

Chugai Pharmaceutical Co. Ltd. (Tokyo:4519), Tokyo, Japan

Daiichi Sankyo Co. Ltd. (Tokyo:4568), Tokyo, Japan

Dana-Farber Cancer Institute, Boston, Mass.

GlaxoSmithKline plc (LSE:GSK; NYSE:GSK), London, U.K.

Harvard Medical School, Boston, Mass.

Japan Tobacco Inc. (Tokyo:2914), Tokyo, Japan

Merck \& Co. Inc. (NYSE:MRK), Whitehouse Station, N.J.

Novartis AG (NYSE:NVS; SIX:NOVN), Basel, Switzerland

Oncothyreon Inc. (NASDAQ:ONTY), Seattle, Wash.

Roche (SIX:ROG; OTCQX:RHHBY), Basel, Switzerland

University of California, Los Angeles, Calif.

The University of Texas MD Anderson Cancer Center, Houston, Texas 